\title{
National Beef Market Basket Survey - 2006: External fat thickness measurements and separable component determinations for beef from US retail establishments
}

\author{
C.L. Mason ${ }^{a}$, K.L. Nicholson ${ }^{\text {a }}$, J.C. Brooks ${ }^{b}$, R.J. Delmore ${ }^{c}$, W.R. Henning ${ }^{\text {d }}$, D.D. Johnson ${ }^{\mathrm{e}}$, \\ C.L. Lorenzen ${ }^{\text {f }}$, R.J. Maddock ${ }^{g}$, R.K. Miller ${ }^{\text {a }}$, J.B. Morgan ${ }^{\text {h }}$, B.E. Wasser ${ }^{i}$, B.L. Gwartney ${ }^{i}$, \\ K.B. Harris ${ }^{\text {a }}$, D.B. Griffin ${ }^{\text {a }}$, D.S. Hale ${ }^{\text {a }}$, J.W. Savell ${ }^{\mathrm{a}, *}$ \\ ${ }^{a}$ Department of Animal Science, Texas AEM University, 2471 TAMU, College Station, TX 77843-2471, USA \\ ${ }^{\mathrm{b}}$ Department of Animal Science, Texas Tech University, P.O. Box 42141, Lubbock, TX 79409, USA \\ ${ }^{\mathrm{c}}$ Department of Animal Science, California Polytechnic State University. Animal Science Bldg 24, San Luis Obispo, CA 93407, USA \\ ${ }^{\mathrm{d}}$ Department of Animal Science, Pennsylvania State University, 0307 AgSci and Ind Bldg, University Park, PA 16802, USA \\ ${ }^{\mathrm{e}}$ Department of Animal Science, University of Florida, P.O. Box 110910, Gainesville, FL 32611, USA \\ ${ }^{\mathrm{f}}$ Department of Food Science, University of Missouri, 256 William C. Stringer Wing, Columbia, MO 65211, USA \\ ${ }^{\mathrm{g}}$ Department of Animal Science, South Dakota State University, 120 AnSci Complex, Brookings, SD 57007, USA \\ h Department of Animal Science, Oklahoma State University, 10413 Animal Science, Stillwater, OK 74078, USA \\ i National Cattlemen's Beef Association, 9110 East Nichols Avenue, Centennial, CO 80112, USA
}

\begin{abstract}
A B S T R A C T
A market basket survey for beef retail cut composition at the retail level (four stores each from two chains in each city) was conducted in 11 US cities from January to March 2006. Beef cuts $(n=17,495)$ were measured for external fat thickness with cuts from the chuck $(0.05 \mathrm{~cm})$, round $(0.05 \mathrm{~cm})$, and miscellaneous $(0.04 \mathrm{~cm})$ having less $(P<0.05)$ fat than cuts from the loin $(0.11 \mathrm{~cm})$ and $\operatorname{rib}(0.11 \mathrm{~cm})$. Beef cuts $(n=1327)$ were separated physically into separable components with round cuts having more $(P<0.05)$ separable lean $(96.63 \%)$ than chuck cuts $(86.81 \%)$ and miscellaneous cuts $(86.18 \%)$, which had more $(P<0.05)$ separable lean than loin cuts $(84.53 \%)$ with rib cuts $(69.34 \%)$ having the lowest $(P<0.05)$ separable lean. Chemical fat from the separable lean differed $(P<0.05)$ between each cut category: round cuts $(3.71 \%)$, miscellaneous cuts $(4.99 \%)$, loin cuts $(5.60 \%)$, chuck cuts $(6.90 \%)$, and rib cuts (8.61\%). Ground beef samples $(n=235$ ), with declared lean/fat percentages ranging from $73 / 27$ to $96 / 4$, had overall chemical fat values of $13.41 \%$ and moisture values of $67.42 \%$. This survey documents the current beef retail cut and ground beef composition, which is helpful to those who need this information for various dietary and marketing purposes.
\end{abstract}

\section{Introduction}

Composition of beef carcasses and cuts has been a long-standing research area for meat scientists throughout the world. The compilation of basic food composition data and the development of composition tables for the United States Department of Agriculture began in the late 1800 s. Since the mid-1900s, continued research in beef composition by universities, agriculture experiment stations, government laboratories, as well as industry, has helped USDA to continue to update and revise nutrient information, resulting in four different versions of the Agriculture Handbook No. 8, "Composition of Foods: Beef Products; Raw, Pro-

\footnotetext{
* Corresponding author. Tel.: +1 979845 3935; fax: +1 9798459454.

E-mail address: j-savell@tamu.edu (J.W. Savell).
}

cessed, Prepared" (commonly referred to in the recent past as Agriculture Handbook 8-13).

Since 1992, beef retail cut composition and nutrient information have been maintained by the United States Department of Agriculture's National Nutrient Data Laboratory through the use of a Nutrient Database for Standard Reference. Several key findings in the beef industry during the late 1980s instigated major changes in the way beef retail cuts have been merchandized and as a consequence made data presented in Agricultural Handbook 8-13 (USDA, 1986) obsolete. The National Consumer Retail Beef Study (Cross, Savell, \& Francis, 1986; Savell et al., 1989) showed that consumers preferred beef retail cuts that were trimmed to have little or no subcutaneous fat. These findings led retailers to reduce fat trim specifications to no more than $0.64 \mathrm{~cm}$ to meet consumer demands (Cross et al., 1986). These results also prompted the need for more research to better define the fatness of beef cuts at the 
retail level and determine to what extent retailers were trimming external fat to meet consumer demands. The National Beef Market Basket Survey (Savell, Harris, Cross, Hale, \& Beasley, 1991) found retail cuts to be even leaner than was expected. Results from this study led to the work by Jones, Savell, and Cross (1992a, 1992b, 1992c) that analyzed the physical and chemical composition of beef retail cuts, raw and cooked, trimmed to $0.0 \mathrm{~cm}$ and $0.6 \mathrm{~cm}$ of external fat. Not only did these data validate the previous reports of leaner beef retail cut composition, but it also was used to update the Agriculture Handbook 8-13 at the time. Regression equations reported in the study could be used to predict the composition of beef retail cuts trimmed to $0.0 \mathrm{~cm}$ and $0.6 \mathrm{~cm}$ external fat regardless of the changes expected in the US beef carcass pop-

\section{Table 1}

Least squares means $\pm \mathrm{SEM}^{\mathrm{A}}$ for external fat thickness for retail cuts from the chuck, rib, loin, and round primals, and other miscellaneous beef cuts surveyed in the retail store

\begin{tabular}{lrl}
\hline & $n$ & External fat thickness $(\mathrm{cm})$ \\
\hline Chuck & 3106 & $0.05 \pm 0.01 \mathrm{~b}$ \\
Rib & 2266 & $0.11 \pm 0.01 \mathrm{c}$ \\
Loin & 4151 & $0.11 \pm 0.004 \mathrm{c}$ \\
Round & 4561 & $0.05 \pm 0.004 \mathrm{ab}$ \\
Miscellaneous & 3411 & $0.04 \pm 0.01 \mathrm{a}$ \\
Total & 17,495 & $P<0.0001$ \\
\hline
\end{tabular}

Means within the same column lacking a common letter $(a-c)$ differ $(P<0.05)$.

a SEM is the standard error of the least squares means.

Table 2

Means and standard deviations (SD) for fat thickness for retail cuts from the chuck and rib surveyed in the retail store

\begin{tabular}{|c|c|c|c|c|}
\hline Approved URMIS a name & $\mathrm{UPC}^{\mathrm{b}}$ & $n$ & Fat thickness $(\mathrm{cm})$ & $\mathrm{SD}$ \\
\hline Chuck & & 3106 & & \\
\hline 7-Bone pot roast & 1033 & 53 & 0.15 & 0.13 \\
\hline 7-Bone steak & 1035 & 31 & 0.11 & 0.08 \\
\hline Arm pot roast & 1048 & 35 & 0.18 & 0.13 \\
\hline Arm pot roast bnls & 1049 & 61 & 0.09 & 0.11 \\
\hline Blade roast & 1064 & 46 & 0.06 & 0.10 \\
\hline Blade steak & 1066 & 61 & 0.07 & 0.08 \\
\hline Blade steak bnls & 1073 & 188 & 0.04 & 0.05 \\
\hline Pot roast bnls & 1080 & 325 & 0.05 & 0.06 \\
\hline Eye steak bnls & 1102 & 145 & 0.04 & 0.05 \\
\hline Flanken style ribs & 1107 & 163 & 0.05 & 0.08 \\
\hline Flanken style ribs bnls & 1110 & 43 & 0.00 & 0.00 \\
\hline Mock tender roast & 1115 & 7 & 0.03 & 0.04 \\
\hline Mock tender steak & 1116 & 116 & 0.01 & 0.03 \\
\hline Neck pot roast bnls & 1121 & 58 & 0.04 & 0.04 \\
\hline Short ribs & 1124 & 337 & 0.05 & 0.10 \\
\hline Short ribs bnls & 1127 & 120 & 0.02 & 0.05 \\
\hline Shoulder pot roast bnls & 1132 & 136 & 0.11 & 0.11 \\
\hline Shoulder steak bnls & 1133 & 326 & 0.10 & 0.07 \\
\hline Shoulder top blade roast bnls & 1137 & 56 & 0.01 & 0.03 \\
\hline Shoulder top blade steak bnls & 1144 & 194 & 0.01 & 0.02 \\
\hline Under blade pot roast & 1150 & 19 & 0.17 & 0.15 \\
\hline Under blade pot roast bnls & 1151 & 280 & 0.05 & 0.06 \\
\hline Under blade steak & 1152 & 7 & 0.05 & 0.07 \\
\hline Under blade skeak bnls & 1158 & 251 & 0.04 & 0.07 \\
\hline Should top blade steak (flat iron) & 1166 & 48 & 0.00 & 0.00 \\
\hline Rib & & 2266 & & \\
\hline Back ribs & 1182 & 219 & 0.01 & 0.02 \\
\hline Ribeye roast & 1192 & 3 & 0.15 & - \\
\hline Ribeye roast lip on BI & 1193 & 24 & 0.14 & 0.06 \\
\hline Ribeye steak lip on BI & 1197 & 544 & 0.13 & 0.09 \\
\hline Ribeye steak lip on bnls & 1203 & 438 & 0.12 & 0.09 \\
\hline Ribeye steak & 1209 & 934 & 0.12 & 0.08 \\
\hline Roast small end & 1235 & 21 & 0.10 & 0.07 \\
\hline Steak small end bnls & 1245 & 83 & 0.14 & 0.08 \\
\hline
\end{tabular}

a Uniform retail meat identity standards (Industry-Wide Cooperative Meat Identification Standards Committee, 2003).

b Universal product code. ulation throughout time. In other research, Wahrmund-Wyle, Harris, and Savell (2000a, 2000b) studied the physical and chemical composition of the separable lean for cuts trimmed to an external fat trim level of $0.6 \mathrm{~cm}$, cooked; $0.3 \mathrm{~cm}$, cooked; $0.3 \mathrm{~cm}$, raw; and

Table 3

Means and standard deviations (SD) for fat thickness for retail cuts from the loin and round surveyed in the retail store

\begin{tabular}{|c|c|c|c|c|}
\hline Approved URMIS ${ }^{\mathrm{a}}$ name & $\mathrm{UPC}^{\mathrm{b}}$ & $n$ & Fat thickness $(\mathrm{cm})$ & $\mathrm{SD}$ \\
\hline Loin & & 4151 & & \\
\hline Ball tip roast & 1307 & 64 & 0.00 & 0.00 \\
\hline Ball tip steak & 1308 & 168 & 0.01 & 0.02 \\
\hline Flap meat steak & 1326 & 5 & 0.00 & 0.00 \\
\hline Porterhouse steak & 1330 & 501 & 0.16 & 0.08 \\
\hline Shell sirloin steak & 1346 & 79 & 0.20 & 0.06 \\
\hline Sirloin steak & 1358 & 14 & 0.17 & 0.15 \\
\hline T-bone steak & 1369 & 629 & 0.15 & 0.08 \\
\hline Tenderloin steak & 1388 & 517 & 0.05 & 0.08 \\
\hline Top loin steak & 1398 & 306 & 0.18 & 0.10 \\
\hline Top loin steak bnls & 1404 & 948 & 0.15 & 0.09 \\
\hline Top sirloin roast bnls cap off & 1419 & 5 & 0.10 & 0.14 \\
\hline Top sirloin cap steak bnls & 1421 & 34 & 0.09 & 0.11 \\
\hline Top sirloin steak bnls & 1422 & 369 & 0.09 & 0.09 \\
\hline Top sirloin steak bnls cap off & 1426 & 401 & 0.07 & 0.08 \\
\hline Tri tip roast & 1429 & 52 & 0.03 & 0.06 \\
\hline Tri tip steak & 1430 & 59 & 0.03 & 0.04 \\
\hline Round & & 4561 & & \\
\hline Top round roast cap off & 1454 & 29 & 0.00 & 0.00 \\
\hline Top round roast & 1455 & 98 & 0.05 & 0.10 \\
\hline Bottom round roast & 1464 & 364 & 0.10 & 0.28 \\
\hline Bottom round steak & 1466 & 523 & 0.07 & 0.07 \\
\hline Eye round roast & 1480 & 249 & 0.06 & 0.09 \\
\hline Eye round steak & 1481 & 443 & 0.04 & 0.06 \\
\hline Round steak & 1494 & 6 & 0.15 & - \\
\hline Round steak bnls & 1501 & 162 & 0.23 & 0.29 \\
\hline Bottom round rump roast & 1519 & 234 & 0.06 & 0.08 \\
\hline Round tip roast & 1525 & 50 & 0.01 & 0.03 \\
\hline Round tip roast cap off & 1526 & 27 & 0.03 & 0.05 \\
\hline Round tip steak & 1527 & 130 & 0.02 & 0.04 \\
\hline Round tip steak cap off & 1535 & 153 & 0.01 & 0.02 \\
\hline Sirloin tip side steak & 1543 & 98 & 0.01 & 0.03 \\
\hline Sirloin tip center roast & 1549 & 6 & 0.00 & 0.00 \\
\hline Sirloin tip center steak & 1550 & 78 & 0.00 & 0.00 \\
\hline Top round steak & 1553 & 674 & 0.04 & 0.08 \\
\hline Top round steak 1 st cut & 1556 & 363 & 0.03 & 0.05 \\
\hline Round for cubed steak & 1577 & 874 & 0.01 & 0.03 \\
\hline
\end{tabular}

a Uniform retail meat identity standards (Industry-Wide Cooperative Meat Identification Standards Committee, 2003).

${ }^{\mathrm{b}}$ Universal product code.

Table 4

Means and standard deviations (SD) for fat thickness for retail cuts from other miscellaneous beef cuts

\begin{tabular}{|c|c|c|c|c|}
\hline Approved URMIS ${ }^{a}$ name & $\mathrm{UPC}^{\mathrm{b}}$ & $n$ & Fat thickness $(\mathrm{cm})$ & SD \\
\hline Miscellaneous & & 3411 & & \\
\hline Flank steak & 1581 & 306 & 0.00 & 0.00 \\
\hline Plate spareribs & 1598 & 15 & - & - \\
\hline Plate short ribs & 1599 & 52 & 0.06 & 0.05 \\
\hline Plate short ribs flaken style & 1603 & 125 & 0.05 & 0.09 \\
\hline Plate short ribs bnls & 1605 & 59 & 0.01 & 0.03 \\
\hline Plate skirt steak bnls & 1607 & 278 & 0.01 & 0.02 \\
\hline Brisket whole bnls & 1615 & 346 & 0.18 & 0.20 \\
\hline Brisket flat cut bnls & 1622 & 252 & 0.10 & 0.14 \\
\hline Brisket edge cut bnls & 1624 & 10 & 0.00 & - \\
\hline Brisket point half bnls & 1628 & 6 & 0.00 & 0.00 \\
\hline Brisket corned bnls & 1630 & 25 & - & - \\
\hline Shank cross cuts & 1636 & 397 & 0.08 & 0.09 \\
\hline Beef for stew & 1727 & 1205 & 0.00 & 0.02 \\
\hline Braising strips & & 21 & 0.02 & 0.04 \\
\hline Stirfry & & 314 & 0.01 & 0.05 \\
\hline
\end{tabular}

${ }^{\text {a }}$ Uniform retail meat identity standards (Industry-Wide Cooperative Meat Identification Standards Committee, 2003).

b Universal product code. 
$0.0 \mathrm{~cm}$, cooked. USDA's nutritional information sources were devoid of this type of information on the separable lean tissue only. Results for chemical fat content from this study for most cuts were lower than what was reported by USDA.

It is apparent that continual work must be done to most accurately represent the ever-changing face of beef retail cut composition. Data presented in the National Nutrient Database are the foundation for a majority of the public and private work in the human nutrition field. Because this information directly impacts nutritional activities within this country, it must be the most accurate and current data available. The federal government not only uses these numbers for various disease and disease treatment research studies, but for dietary guidance and the planning of national nutritional policies as well. The objective of this study was to collect external fat thickness measurements, conduct separable component, and perform chemical fat analyses on retail cuts to gain knowledge of the composition of retail raw beef throughout the United States.

Table 5

Means and standard deviations (SD) for percentage separable components of retail cuts from the beef chuck

\begin{tabular}{|c|c|c|c|c|c|c|c|c|c|c|c|c|}
\hline \multirow[t]{2}{*}{ Approved URMIS ${ }^{\text {a }}$ name } & \multirow[t]{2}{*}{$\mathrm{UPC}^{\mathrm{b}}$} & \multirow[t]{2}{*}{$n$} & \multicolumn{2}{|c|}{ Lean $(\%)$} & \multicolumn{2}{|c|}{ External fat (\%) } & \multicolumn{2}{|c|}{ Seam fat (\%) } & \multicolumn{2}{|c|}{ Total fat (\%) } & \multicolumn{2}{|c|}{ Bone and connective tissue (\%) } \\
\hline & & & Mean & SD & Mean & SD & Mean & SD & Mean & SD & Mean & SD \\
\hline \multicolumn{13}{|l|}{ Beef chuck } \\
\hline Arm pot roast & 1048 & 3 & 73.34 & 7.12 & 3.88 & 3.51 & 7.43 & 0.87 & 11.31 & 3.30 & 15.59 & 4.82 \\
\hline Arm pot roast bnls & 1049 & 3 & 85.42 & 11.11 & 4.17 & 7.22 & 9.56 & 2.41 & 13.73 & 9.63 & 0.85 & 1.47 \\
\hline Shoulder pot roast bnls & 1132 & 27 & 88.97 & 5.37 & 5.59 & 3.14 & 3.17 & 3.33 & 8.76 & 4.32 & 2.27 & 3.02 \\
\hline Arm steak bnls & 1056 & 1 & 94.59 & & 0.70 & & 3.14 & & 3.84 & & 1.57 & \\
\hline Short ribs & 1124 & 32 & 55.90 & 14.84 & 7.77 & 6.03 & 5.73 & 4.92 & 13.50 & 6.79 & 30.59 & 11.98 \\
\hline Short ribs bnls & 1127 & 8 & 94.30 & 5.89 & 4.25 & 4.70 & 1.21 & 2.14 & 5.46 & 6.10 & 0.24 & 0.68 \\
\hline Shoulder steak bnls & 1133 & 66 & 93.58 & 4.14 & 3.62 & 2.93 & 1.22 & 1.76 & 4.84 & 3.75 & 1.53 & 2.24 \\
\hline Flanken style ribs & 1107 & 9 & 60.10 & 5.53 & 5.46 & 3.60 & 4.62 & 3.15 & 10.08 & 4.39 & 29.82 & 5.08 \\
\hline Flanken style ribs bnls & 1110 & 1 & 86.61 & & 5.29 & & 6.45 & & 11.74 & & 1.65 & \\
\hline Neck pot roast bnls & 1121 & 6 & 86.15 & 3.85 & 4.93 & 4.71 & 5.41 & 2.04 & 10.34 & 3.54 & 3.51 & 2.66 \\
\hline Pot roast bnls & 1080 & 22 & 86.35 & 3.53 & 2.62 & 2.13 & 9.26 & 3.54 & 11.88 & 3.75 & 1.71 & 2.14 \\
\hline 7-bone pot roast & 1033 & 4 & 65.00 & 5.90 & 3.47 & 2.27 & 9.49 & 0.90 & 12.96 & 2.28 & 22.04 & 4.27 \\
\hline 7-bone steak & 1035 & 6 & 65.03 & 3.47 & 3.70 & 1.72 & 12.44 & 4.15 & 16.14 & 4.50 & 18.84 & 1.92 \\
\hline Blade roast & 1064 & 4 & 60.95 & 1.24 & 4.33 & 3.52 & 10.59 & 4.24 & 14.92 & 3.46 & 24.13 & 3.70 \\
\hline Blade steak & 1066 & 5 & 63.77 & 2.15 & 2.54 & 1.09 & 16.49 & 3.59 & 19.03 & 4.05 & 17.20 & 3.43 \\
\hline Blade steak bnls & 1073 & 27 & 88.50 & 3.48 & 1.53 & 1.85 & 8.94 & 4.21 & 10.47 & 3.77 & 1.04 & 1.19 \\
\hline Top blade steak BI & 1138 & 4 & 69.50 & 16.35 & 13.66 & 22.11 & 1.81 & 2.78 & 15.47 & 20.75 & 15.03 & 4.81 \\
\hline Under blade pot roast & 1150 & 1 & 86.21 & & 0.00 & & 9.29 & & 9.29 & & 4.50 & \\
\hline Under blade steak & 1152 & 5 & 82.30 & 6.86 & 1.85 & 1.70 & 8.54 & 3.72 & 10.39 & 4.21 & 7.32 & 9.10 \\
\hline Under blade pot roast bnls & 1151 & 21 & 81.72 & 4.82 & 2.26 & 2.64 & 13.96 & 4.11 & 16.22 & 4.27 & 2.06 & 1.66 \\
\hline Under blade steak bnls & 1158 & 23 & 86.19 & 5.83 & 1.61 & 1.56 & 10.21 & 5.54 & 11.82 & 6.16 & 1.81 & 2.76 \\
\hline Mock tender roast & 1115 & 3 & 97.40 & 0.28 & 0.66 & 0.73 & 0.49 & 0.44 & 1.15 & 0.29 & 1.45 & 0.11 \\
\hline Mock tender steak & 1116 & 62 & 97.54 & 3.33 & 1.09 & 2.27 & 0.06 & 0.29 & 1.14 & 2.26 & 1.28 & 2.57 \\
\hline Top blade roast bnls & 1137 & 15 & 94.29 & 5.75 & 2.17 & 4.46 & 0.17 & 0.45 & 2.34 & 4.40 & 3.37 & 3.51 \\
\hline Top blade steak bnls & 1144 & 110 & 97.85 & 4.03 & 0.29 & 0.84 & 0.33 & 1.82 & 0.62 & 1.96 & 1.54 & 3.43 \\
\hline Top blade steak (flat iron) & 1166 & 7 & 97.87 & 2.44 & 0.92 & 1.17 & 0.00 & 0.00 & 0.92 & 1.17 & 1.21 & 2.02 \\
\hline Eye steak bnls & 1102 & 66 & 83.20 & 6.90 & 4.33 & 3.40 & 9.94 & 4.74 & 14.27 & 5.32 & 2.53 & 4.19 \\
\hline Chuck average & & 541 & 87.81 & 13.25 & 2.81 & 4.00 & 4.56 & 5.61 & 7.37 & 7.18 & 4.81 & 9.24 \\
\hline
\end{tabular}

a Uniform retail meat identity standards (Industry-Wide Cooperative Meat Identification Standards Committee, 2003).

b Universal product code.

Table 6

Means and standard deviations (SD) for percentage separable components of retail cuts from the beef rib

\begin{tabular}{|c|c|c|c|c|c|c|c|c|c|c|c|c|}
\hline \multirow[t]{2}{*}{ Approved URMIS ${ }^{\mathrm{a}}$ name } & \multirow[t]{2}{*}{$\mathrm{UPC}^{\mathrm{b}}$} & \multirow[t]{2}{*}{$n$} & \multicolumn{2}{|c|}{ Lean $(\%)$} & \multicolumn{2}{|c|}{ External fat (\%) } & \multicolumn{2}{|c|}{ Seam fat (\%) } & \multicolumn{2}{|c|}{ Total fat (\%) } & \multicolumn{2}{|c|}{ Bone and connective tissue (\%) } \\
\hline & & & Mean & SD & Mean & SD & Mean & SD & Mean & SD & Mean & SD \\
\hline \multicolumn{13}{|l|}{ Beef rib } \\
\hline Roast large end & 1218 & 3 & 67.97 & 2.24 & 7.14 & 3.13 & 9.68 & 1.05 & 16.82 & 2.87 & 15.21 & 0.65 \\
\hline Steak large end & 1222 & 3 & 72.20 & 10.46 & 10.23 & 4.90 & 12.37 & 4.78 & 22.60 & 9.43 & 5.20 & 3.11 \\
\hline Back ribs & 1182 & 34 & 34.45 & 6.20 & 9.27 & 5.36 & 10.22 & 5.86 & 19.49 & 7.23 & 46.06 & 7.98 \\
\hline Roast small end & 1235 & 4 & 68.57 & 3.53 & 7.94 & 3.63 & 8.88 & 4.19 & 16.82 & 2.34 & 14.60 & 3.71 \\
\hline Roast small end bnls & 1238 & 1 & 68.82 & & 5.69 & & 11.82 & & 17.51 & & 13.68 & \\
\hline Steak small end & 1239 & 5 & 71.93 & 6.73 & 7.97 & 4.66 & 8.35 & 2.61 & 16.32 & 2.49 & 11.75 & 8.23 \\
\hline Steak small end bnls & 1245 & 8 & 78.02 & 5.74 & 8.12 & 2.93 & 8.04 & 3.50 & 16.16 & 3.56 & 5.81 & 3.67 \\
\hline Ribeye steak & 1209 & 62 & 80.56 & 6.09 & 6.85 & 4.86 & 10.54 & 5.38 & 17.39 & 6.68 & 2.04 & 2.15 \\
\hline Ribeye roast & 1192 & 1 & 61.99 & & 3.78 & & 22.97 & & 26.75 & & 11.25 & \\
\hline Short ribs & 1259 & 3 & 54.48 & 7.46 & 5.81 & 6.33 & 9.60 & 5.00 & 15.41 & 5.19 & 30.10 & 3.50 \\
\hline Short ribs bnls & 1265 & 1 & 85.74 & & 14.26 & & 0.00 & & 14.26 & & 0.00 & \\
\hline Ribeye rst lip on BI & 1193 & 7 & 69.45 & 4.69 & 8.68 & 2.64 & 10.43 & 3.72 & 19.11 & 3.65 & 11.44 & 1.66 \\
\hline Ribeye stk lip on BI & 1197 & 47 & 68.58 & 7.75 & 6.75 & 3.79 & 10.57 & 4.55 & 17.32 & 4.89 & 14.10 & 6.85 \\
\hline Ribeye stk lip on bnls & 1203 & 49 & 79.10 & 4.55 & 6.69 & 3.58 & 11.36 & 4.76 & 18.05 & 5.40 & 2.85 & 2.97 \\
\hline Rib average & & 228 & 69.34 & 16.76 & 7.35 & 4.37 & 10.52 & 5.01 & 17.87 & 5.84 & 12.79 & 15.82 \\
\hline
\end{tabular}

a Uniform retail meat identity standards (Industry-Wide Cooperative Meat Identification Standards Committee, 2003).

b Universal product code. 


\section{Materials and methods}

\subsection{Product selection}

Eleven cities were selected to allow sampling in various geographical regions of the United States with known differences in market preference. Cities sampled included: New York, NY; Philadelphia, PA; Atlanta, GA; Chicago, IL; Kansas City, MO; Houston, TX; Denver, CO; Los Angeles, CA; San Francisco, CA; Seattle, WA; and Tampa, FL. Cities were chosen to allow for comparison to the previous market basket survey and to provide additional opportunities for data collection. Two retail chains per city were selected with the chains representing at least one third of the total volume of supermarket sales in that city. Four stores per chain were chosen so that a total of eight supermarket stores per metropolitan area were sampled. Sampling occurred in the months of January to March 2006.

At the store level, external fat thickness, when present, was measured on all steaks and roasts at three different locations on the cut. These measurements were used to calculate an average external fat thickness measurement for each cut. Those cuts that were free of external fat were noted as such. Additional information that appeared on the meat label or package also was recorded. After all measurements and data were collected at the store, an assortment of 21 retail cuts, representing various locations of the carcass, were purchased from each store and shipped to the Rosenthal Meat Science and Technology Center at Texas A\&M University for cut dissection and chemical fat determination. Cuts from the

Table 7

Means and standard deviations (SD) for percentage separable components of retail cuts from the beef loin

\begin{tabular}{|c|c|c|c|c|c|c|c|c|c|c|c|c|}
\hline \multirow[t]{2}{*}{ Approved URMIS ${ }^{\mathrm{a}}$ name } & \multirow[t]{2}{*}{$\mathrm{UPC}^{\mathrm{b}}$} & \multirow[t]{2}{*}{$n$} & \multicolumn{2}{|c|}{ Lean $(\%)$} & \multicolumn{2}{|c|}{ External fat (\%) } & \multicolumn{2}{|c|}{ Seam fat (\%) } & \multicolumn{2}{|c|}{ Total fat (\%) } & \multicolumn{2}{|c|}{ Bone and connective tissue (\%) } \\
\hline & & & Mean & SD & Mean & SD & Mean & SD & Mean & SD & Mean & SD \\
\hline \multicolumn{13}{|l|}{ Beef loin } \\
\hline Top loin steak & 1398 & 24 & 68.06 & 7.15 & 7.07 & 3.86 & 5.02 & 4.82 & 12.09 & 5.59 & 19.88 & 7.11 \\
\hline Top loin steak bnls & 1404 & 80 & 83.29 & 5.38 & 10.08 & 6.85 & 3.35 & 3.62 & 13.43 & 7.30 & 3.77 & 3.66 \\
\hline Tenderloin steak & 1388 & 66 & 92.33 & 6.66 & 3.20 & 2.69 & 2.01 & 3.47 & 5.21 & 4.70 & 2.46 & 5.07 \\
\hline T-bone steak & 1369 & 49 & 66.56 & 7.30 & 6.56 & 3.18 & 5.55 & 5.28 & 12.11 & 5.89 & 21.74 & 6.61 \\
\hline Porterhouse steak & 1330 & 21 & 69.93 & 4.64 & 6.61 & 4.26 & 6.36 & 4.37 & 12.97 & 4.26 & 17.1 & 3.92 \\
\hline Sirloin steak & 1358 & 1 & 78.32 & & 5.08 & & 8.40 & & 13.48 & & 9.18 & \\
\hline Shell sirloin steak & 1346 & 1 & 75.69 & & 1.75 & & 10.53 & & 12.28 & & 12.03 & \\
\hline Ball tip roast & 1307 & 6 & 92.61 & 3.85 & 4.82 & 3.42 & 1.13 & 1.68 & 5.95 & 3.61 & 1.72 & 3.45 \\
\hline Ball tip steak & 1308 & 22 & 95.83 & 3.30 & 2.29 & 2.85 & 1.30 & 1.79 & 3.59 & 3.10 & 0.58 & 1.37 \\
\hline Flap meat steak & 1326 & 1 & 90.19 & & 6.70 & & 0.96 & & 7.66 & & 2.15 & \\
\hline Tri tip roast & 1429 & 12 & 88.37 & 9.32 & 10.87 & 8.66 & 0.38 & 1.08 & 11.25 & 9.02 & 0.38 & 0.68 \\
\hline Tri tip steak & 1430 & 26 & 92.29 & 6.31 & 6.58 & 5.81 & 0.40 & 1.51 & 6.98 & 6.98 & 0.73 & 1.95 \\
\hline Top sirloin rst bnls cap off & 1419 & 1 & 90.43 & & 8.56 & & 0.00 & & 8.56 & & 1.01 & \\
\hline Top sirloin steak bnls & 1422 & 26 & 90.70 & 4.12 & 4.74 & 3.29 & 3.27 & 2.99 & 8.01 & 3.90 & 1.29 & 1.83 \\
\hline Top sirloin stk bnls cap off & 1426 & 33 & 94.15 & 3.77 & 3.12 & 2.79 & 1.54 & 2.13 & 4.66 & 3.04 & 1.19 & 1.86 \\
\hline Top sirloin cap steak bnls & 1421 & 13 & 95.81 & 4.94 & 3.31 & 4.78 & 0.00 & 0.00 & 3.31 & 4.78 & 0.71 & 0.93 \\
\hline Loin average & & 382 & 84.53 & 11.96 & 6.07 & 5.37 & 2.97 & 3.95 & 9.04 & 6.70 & 6.59 & 8.99 \\
\hline
\end{tabular}

a Uniform retail meat identity standards (Industry-Wide Cooperative Meat Identification Standards Committee, 2003).

b Universal product code.

Table 8

Means and standard deviations (SD) for percentage separable components of retail cuts from the beef round

\begin{tabular}{|c|c|c|c|c|c|c|c|c|c|c|c|c|}
\hline \multirow[t]{2}{*}{ Approved URMIS ${ }^{a}$ name } & \multirow[t]{2}{*}{$\mathrm{UPC}^{\mathrm{b}}$} & \multirow[t]{2}{*}{$n$} & \multicolumn{2}{|c|}{ Lean $(\%)$} & \multicolumn{2}{|c|}{ External fat (\%) } & \multicolumn{2}{|c|}{ Seam fat (\%) } & \multicolumn{2}{|c|}{ Total fat (\%) } & \multicolumn{2}{|c|}{ Bone and connective tissue (\%) } \\
\hline & & & Mean & SD & Mean & SD & Mean & SD & Mean & SD & Mean & SD \\
\hline \multicolumn{13}{|l|}{ Beef round } \\
\hline Steak & 1494 & 1 & 86.07 & & 6.21 & & 4.08 & & 10.29 & & 3.64 & \\
\hline Steak bnls & 1501 & 15 & 89.55 & 3.44 & 4.95 & 2.25 & 4.51 & 2.32 & 9.46 & 3.90 & 1.00 & 0.95 \\
\hline Top round roast & 1455 & 6 & 91.61 & 3.45 & 2.41 & 2.16 & 4.19 & 2.85 & 6.60 & 2.62 & 1.79 & 1.98 \\
\hline Top round roast cap off & 1454 & 7 & 98.34 & 1.50 & 1.34 & 1.15 & 0.32 & 0.84 & 1.66 & 1.50 & 0.00 & 0.00 \\
\hline Top round steak, 1 st cut & 1556 & 6 & 91.25 & 18.05 & 8.43 & 18.21 & 0.30 & 0.75 & 8.73 & 18.06 & 0.02 & 0.05 \\
\hline Top round steak & 1553 & 40 & 97.43 & 3.09 & 1.60 & 2.22 & 0.91 & 1.71 & 2.51 & 3.08 & 0.07 & 0.42 \\
\hline Bottom round rump roast & 1519 & 20 & 93.77 & 3.84 & 5.23 & 3.53 & 0.52 & 0.85 & 5.75 & 4.02 & 0.48 & 1.29 \\
\hline Bottom round roast & 1464 & 22 & 91.81 & 4.25 & 5.91 & 3.37 & 1.17 & 1.68 & 7.08 & 4.35 & 1.11 & 1.31 \\
\hline Bottom round steak & 1466 & 55 & 95.57 & 3.52 & 3.28 & 3.48 & 0.58 & 1.27 & 3.86 & 3.40 & 0.58 & 1.29 \\
\hline Eye round roast & 1480 & 24 & 95.46 & 3.67 & 3.88 & 2.94 & 0.39 & 1.00 & 4.27 & 3.74 & 0.27 & 0.58 \\
\hline Eye round steak & 1481 & 79 & 98.01 & 2.31 & 1.71 & 2.24 & 0.02 & 0.11 & 1.73 & 2.23 & 0.27 & 0.83 \\
\hline Bottom round rst (triangle) & 1463 & 2 & 93.59 & 0.87 & 6.41 & 0.87 & 0.00 & 0.00 & 6.41 & 0.87 & 0.00 & 0.00 \\
\hline Tip roast & 1525 & 5 & 93.95 & 2.63 & 1.09 & 0.65 & 3.35 & 1.94 & 4.44 & 2.28 & 1.62 & 1.54 \\
\hline Tip steak & 1527 & 16 & 95.88 & 3.96 & 1.74 & 2.64 & 1.58 & 2.17 & 3.32 & 3.23 & 0.79 & 1.50 \\
\hline Tip roast cap off & 1526 & 2 & 97.86 & 3.03 & 0.52 & 0.73 & 1.62 & 2.30 & 2.14 & 3.03 & 0.00 & 0.00 \\
\hline Tip steak cap off & 1535 & 39 & 98.94 & 1.95 & 0.41 & 0.75 & 0.16 & 0.53 & 0.57 & 1.02 & 0.50 & 1.42 \\
\hline Sirloin tip center steak & 1550 & 10 & 98.58 & 1.49 & 0.99 & 0.91 & 0.00 & 0.00 & 0.99 & 0.91 & 0.43 & 1.26 \\
\hline Sirloin tip center roast & 1549 & 4 & 94.31 & 2.84 & 2.98 & 2.18 & 1.18 & 1.46 & 4.16 & 3.51 & 1.53 & 3.06 \\
\hline Sirloin tip side steak & 1543 & 16 & 99.38 & 1.32 & 0.35 & 0.64 & 0.21 & 0.82 & 0.56 & 1.33 & 0.07 & 0.28 \\
\hline Cubed steak & 1577 & 53 & 99.63 & 1.18 & 0.15 & 0.41 & 0.22 & 1.14 & 0.37 & 1.18 & 0.00 & 0.00 \\
\hline Round average & & 422 & 96.63 & 4.34 & 2.27 & 3.56 & 0.68 & 1.57 & 2.96 & 4.12 & 0.42 & 1.09 \\
\hline
\end{tabular}

a Uniform retail meat identity standards (Industry-Wide Cooperative Meat Identification Standards Committee, 2003)

b Universal product code. 
following primals or sections were selected for dissection studies: chuck blade section, chuck arm section, rib, loin, round, and miscellaneous (e.g., stew meat, stirfry, or skirt steak). Ground beef samples were obtained for chemical fat and moisture analyses. Packages were shipped in plastic coolers or insulated boxes the same day for overnight delivery.

\subsection{Retail cut dissection}

Upon arrival, retail cuts were identified according to the uniform retail meat identity standards (URMIS) with both the official URMIS name and UPC code (Industry-Wide Cooperative Meat Identification Standards Committee, 2003). Cuts were removed from the package and dissected into separable lean, external fat (which may have included subcutaneous or intermuscular fat, depending on where the cut was fabricated from the carcass), seam (intermuscular) fat, and bone and heavy connective tissue (waste). Heavy connective tissue within muscles (e.g., Top Blade Steaks or the Muscularis infraspinatus) was not removed; however, heavy connective tissue between muscles was removed. Initial cut weight and post-dissection weights of all components were taken to ascertain the percentages of each dissected component for that cut. Dissection data were used later to determine the fatness of retail cuts. For those packages containing multiple steaks, each steak was weighed and treated independently during dissection, but separable lean was combined for powdering before chemical analysis.

\subsection{Sample preparation}

Separable lean from all steaks and roasts from each package were powdered to make a homogenous sample for chemical fat analysis. Powdering occurred immediately after dissection and weighing. Separable lean from each cut was submerged in liquid nitrogen and then placed in stainless steel blending cups to powder. Two Whirl-Pak bags per retail cut were filled with the resulting powdered sample and stored at $-10{ }^{\circ} \mathrm{C}$ until used for chemical fat analysis.

Ground beef samples, stew meat, cubed meat, stirfry, or any other cuts that had no visible external or seam fat to remove were immediately weighed and powdered. As stated earlier, for those packages containing multiple steaks, each steak was weighed and treated independently during dissection; however, the entire package was combined as a composite for powdering. Cuts that were very large (e.g., whole briskets) were dissected into separable components with all appropriate weights collected and separable lean was sent through a small table-top grinder for homogenization. Smaller grab samples were taken from each quadrant of this homogenized separable lean for powdering.

\subsection{Chemical fat analysis}

Chemical fat of the separable lean from each package, as well as ground beef samples, were measured using a modified version of the oven-dry ether extraction method described by AOAC (2000).

Table 9

Means and standard deviations (SD) for percentage separable components of miscellaneous beef retail cuts

\begin{tabular}{|c|c|c|c|c|c|c|c|c|c|c|c|c|}
\hline \multirow[t]{2}{*}{ Approved URMIS ${ }^{\mathrm{a}}$ name } & \multirow[t]{2}{*}{$\mathrm{UPC}^{\mathrm{b}}$} & \multirow[t]{2}{*}{$n$} & \multicolumn{2}{|c|}{ Lean $(\%)$} & \multicolumn{2}{|c|}{ External fat (\%) } & \multicolumn{2}{|c|}{ Seam fat (\%) } & \multicolumn{2}{|c|}{ Total fat (\%) } & \multicolumn{2}{|c|}{ Bone and connective tissue (\%) } \\
\hline & & & Mean & SD & Mean & SD & Mean & SD & Mean & SD & Mean & SD \\
\hline $\begin{array}{l}\text { Beef shank cross cuts } \\
\text { Beef Brisket }\end{array}$ & 1636 & 58 & 58.66 & 14.79 & 3.77 & 5.32 & 2.99 & 3.16 & 6.76 & 6.48 & 34.65 & 12.31 \\
\hline Whole bnls & 1615 & 3 & 71.83 & 14.95 & 15.50 & 6.87 & 12.67 & 11.72 & 28.17 & 14.95 & 0.00 & 0.00 \\
\hline Point half bnls & 1628 & 1 & 87.26 & & 12.74 & & 0.00 & & 12.74 & & 0.00 & \\
\hline Flat half bnls & 1623 & 14 & 86.15 & 5.33 & 12.68 & 5.49 & 1.17 & 3.16 & 13.85 & 5.33 & 0.00 & 0.00 \\
\hline Middle cut bnls & 1626 & 2 & 77.47 & 3.29 & 18.37 & 1.69 & 4.16 & 4.99 & 22.53 & 3.29 & 0.00 & 0.00 \\
\hline Flat cut bnls & 1622 & 18 & 91.88 & 7.14 & 7.04 & 7.12 & 0.45 & 1.12 & 7.49 & 7.25 & 0.63 & 1.92 \\
\hline Point off bnls & 1629 & 5 & 89.65 & 3.37 & 9.94 & 3.54 & 0.42 & 0.94 & 10.36 & 3.37 & 0.00 & 0.00 \\
\hline Edge cut bnls & 1624 & 1 & 78.05 & & 5.91 & & 16.04 & & 21.95 & & 0.00 & \\
\hline $\begin{array}{l}\text { Beef flank steak } \\
\text { Beef Plate }\end{array}$ & 1581 & 38 & 98.20 & 1.65 & 1.46 & 1.55 & 0.16 & 0.67 & 1.62 & 1.54 & 0.18 & 0.46 \\
\hline Skirt steak bnls & 1607 & 25 & 89.91 & 12.13 & 6.81 & 9.27 & 0.60 & 2.48 & 7.41 & 9.14 & 2.71 & 9.79 \\
\hline Skirt steak rolls bnls & 1611 & 10 & 100.00 & 0.00 & 0.00 & 0.00 & 0.00 & 0.00 & 0.00 & 0.00 & 0.00 & 0.00 \\
\hline Short ribs & 1599 & 7 & 47.49 & 9.99 & 8.69 & 6.04 & 10.52 & 6.56 & 19.21 & 8.32 & 33.29 & 6.82 \\
\hline Spareribs & 1598 & 1 & 37.86 & & 8.95 & & 5.61 & & 14.56 & & 47.57 & \\
\hline Short ribs bnls & 1605 & 8 & 90.62 & 6.12 & 4.06 & 4.77 & 2.07 & 3.70 & 6.13 & 6.16 & 3.25 & 4.09 \\
\hline Short ribs flanken style & 1603 & 10 & 62.07 & 6.33 & 3.36 & 3.61 & 8.25 & 7.24 & 11.61 & 6.07 & 26.32 & 4.04 \\
\hline Beef for stew & 1727 & 43 & 96.45 & 13.9 & 1.11 & 3.05 & 0.24 & 1.09 & 1.35 & 3.28 & 0.11 & 0.39 \\
\hline Beef for stirfry & & 30 & 99.98 & 0.13 & 0.02 & 0.13 & 0.00 & 0.00 & 0.02 & 0.13 & 0.00 & 0.00 \\
\hline Miscellaneous average & & 273 & 84.23 & 19.95 & 3.97 & 6.05 & 1.73 & 3.88 & 5.65 & 7.60 & 9.75 & 16.31 \\
\hline
\end{tabular}

${ }^{\text {a }}$ Uniform retail meat identity standards (Industry-Wide Cooperative Meat Identification Standards Committee, 2003)

b Universal product code.

Table 10

Least squares means $\pm \mathrm{SEM}^{\mathrm{a}}$ for separable components of retail cuts from the chuck, rib, loin, and round primals, and other miscellaneous beef cuts

\begin{tabular}{|c|c|c|c|c|c|c|}
\hline Percentage & Chuck & Rib & Loin & Round & Misc & $P>F$ \\
\hline Lean & $86.81 \pm 0.56 b$ & $69.34 \pm 0.89 d$ & $84.53 \pm 0.69 c$ & $96.63 \pm 0.65 a$ & $86.18 \pm 0.85 b c$ & $<0.0001$ \\
\hline External fat & $2.92 \pm 0.19 d$ & $7.35 \pm 0.31 \mathrm{a}$ & $6.07 \pm 0.24 b$ & $2.27 \pm 0.22 \mathrm{e}$ & $3.82 \pm 0.29 c$ & $<0.0001$ \\
\hline Seam fat & $4.67 \pm 0.18 b$ & $10.52 \pm 0.28 a$ & $2.97 \pm 0.22 c$ & $0.68 \pm 0.21 d$ & $1.18 \pm 0.27 d$ & $<0.0001$ \\
\hline Total fat & $7.56 \pm 0.27 c$ & $17.87 \pm 0.42 a$ & $9.04 \pm 0.33 b$ & $2.96 \pm 0.31 \mathrm{e}$ & $5.00 \pm 0.41 d$ & $<0.0001$ \\
\hline Bone and connective tissue & $5.59 \pm 0.45 c$ & $12.79 \pm 0.71 \mathrm{a}$ & $6.59 \pm 0.55 c$ & $0.42 \pm 0.52 \mathrm{~d}$ & $8.47 \pm 0.68 b$ & $<0.0001$ \\
\hline
\end{tabular}

Means within the same row lacking a common letter (a-e) differ $P<0.05$

a SEM is the standard error for least squares means. 


\subsection{Statistical analysis}

Means, standard deviations, and percentage values were computed using data analysis functions in Microsoft Excel (Microsoft Corporation, Redmond, Washington). Least squares means were separated using PROC GLM with pdiff option (SAS Institute, Cary, North Carolina).

\section{Results and discussion}

\subsection{Store data}

External fat thickness measurements for cuts surveyed in the retail store are presented in Table 1 . When comparing cuts originating from one of the four main primals, cuts from the round,

Table 11

Means and standard deviations for percentage extractable fat and moisture (separable lean only) for retail cuts from the beef chuck

\begin{tabular}{|c|c|c|c|c|c|c|}
\hline \multirow[t]{2}{*}{ Approved URMIS ${ }^{a}$ name } & \multirow[t]{2}{*}{$\mathrm{UPC}^{\mathrm{b}}$} & \multirow[t]{2}{*}{$n$} & \multicolumn{2}{|c|}{ Extractable fat (\%) } & \multicolumn{2}{|c|}{ Moisture (\%) } \\
\hline & & & Mean & SD & Mean & SD \\
\hline \multicolumn{7}{|l|}{ Beef chuck } \\
\hline Arm pot roast & 1048 & 3 & 3.35 & 0.74 & 75.47 & 0.78 \\
\hline Arm pot roast bnls & 1049 & 3 & 4.64 & 1.85 & 74.13 & 1.35 \\
\hline Shoulder pot roast bnls & 1132 & 27 & 3.96 & 1.22 & 74.32 & 1.34 \\
\hline Arm steak bnls & 1056 & 1 & 3.10 & & 74.95 & \\
\hline Short ribs & 1124 & 23 & 10.22 & 4.56 & 70.28 & 3.65 \\
\hline Short ribs bnls & 1127 & 6 & 8.40 & 4.98 & 71.34 & 3.43 \\
\hline Shoulder steak bnls & 1133 & 38 & 4.35 & 1.23 & 73.73 & 1.30 \\
\hline Flanken style ribs & 1107 & 9 & 10.31 & 1.77 & 70.47 & 1.42 \\
\hline Flanken style ribs bnls & 1110 & 1 & 7.45 & & 70.87 & \\
\hline Neck pot roast bnls & 1121 & 6 & 4.99 & 2.14 & 73.86 & 1.72 \\
\hline Pot roast bnls & 1080 & 21 & 6.30 & 2.16 & 72.93 & 1.65 \\
\hline 7-bone pot roast & 1033 & 4 & 7.40 & 2.93 & 72.32 & 2.63 \\
\hline 7-bone steak & 1035 & 6 & 7.48 & 2.44 & 72.15 & 2.11 \\
\hline Blade roast & 1064 & 4 & 7.92 & 1.59 & 71.93 & 1.44 \\
\hline Blade steak & 1066 & 5 & 9.61 & 1.63 & 69.93 & 1.12 \\
\hline Blade steak bnls & 1073 & 24 & 5.58 & 2.25 & 73.41 & 1.90 \\
\hline Top blade steak BI & 1138 & 2 & 7.77 & 0.24 & 72.03 & 0.24 \\
\hline Under blade pot roast & 1150 & 1 & 4.77 & & 73.79 & \\
\hline Under blade steak & 1152 & 3 & 7 & 0.44 & 72.04 & 0.52 \\
\hline Under blade pot roast bnls & 1151 & 20 & 7.55 & 1.82 & 71.97 & 1.54 \\
\hline Under blade steak bnls & 1158 & 19 & 6.41 & 2.57 & 72.76 & 2.22 \\
\hline Mock tender roast & 1115 & 3 & 3.65 & 1.08 & 74.45 & 0.88 \\
\hline Mock tender steak & 1116 & 19 & 3.23 & 1.58 & 75.27 & 1.44 \\
\hline Top blade roast bnls & 1137 & 14 & 6.95 & 1.65 & 72.66 & 1.47 \\
\hline Top blade steak bnls & 1144 & 28 & 7.32 & 2.70 & 72.10 & 2.15 \\
\hline Top blade steak (flat iron) & 1166 & 8 & 7.88 & 2.11 & 72.25 & 1.83 \\
\hline Eye steak bnls & 1102 & 32 & 8.92 & 2.53 & 70.41 & 2.19 \\
\hline Chuck average & & 330 & 6.58 & 3.10 & 72.59 & 2.41 \\
\hline
\end{tabular}

${ }^{a}$ Uniform retail meat identity standards (Industry-Wide Cooperative Meat Identification Standards Committee, 2003)

b Universal product code.

Table 12

Means and standard deviations for percentage extractable fat and moisture (separable lean only) for retail cuts from the beef rib

\begin{tabular}{|c|c|c|c|c|c|c|}
\hline \multirow[t]{2}{*}{ Approved URMIS ${ }^{\mathrm{a}}$ name } & \multirow[t]{2}{*}{$\mathrm{UPC}^{\mathrm{b}}$} & \multirow[t]{2}{*}{$n$} & \multicolumn{2}{|c|}{ Extractable fat (\%) } & \multicolumn{2}{|c|}{ Moisture (\%) } \\
\hline & & & Mean & SD & Mean & SD \\
\hline \multicolumn{7}{|l|}{ Beef rib } \\
\hline Roast large end & 1218 & 3 & 9.15 & 2.81 & 70.59 & 2.36 \\
\hline Steak large end & 1222 & 3 & 8.74 & 1.00 & 69.92 & 1.51 \\
\hline Back ribs & 1182 & 33 & 11.67 & 3.48 & 67.81 & 2.85 \\
\hline Roast small end & 1235 & 4 & 8.53 & 2.73 & 70.25 & 2.16 \\
\hline Roast small end bnls & 1238 & 1 & 9.02 & & 70.08 & \\
\hline Steak small end & 1239 & 4 & 8.62 & 2.45 & 69.72 & 2.24 \\
\hline Steak small end bnls & 1245 & 7 & 7.09 & 2.71 & 71.06 & 1.84 \\
\hline Ribeye steak & 1209 & 46 & 7.97 & 3.18 & 70.61 & 2.46 \\
\hline Ribeye roast & 1192 & 1 & 12.55 & & 67.63 & \\
\hline Short ribs & 1259 & 3 & 11.45 & 3.04 & 69.25 & 1.65 \\
\hline Short ribs bnls & 1265 & 1 & 8.49 & & 70.79 & \\
\hline Ribeye rst lip on BI & 1193 & 7 & 7.75 & 2.28 & 70.58 & 1.62 \\
\hline Ribeye stk lip on BI & 1197 & 45 & 7.58 & 2.59 & 70.63 & 1.92 \\
\hline Ribeye stk lip on bnls & 1203 & 39 & 8.02 & 2.80 & 70.21 & 2.32 \\
\hline Rib average & & 197 & 8.61 & 3.23 & 70.00 & 2.47 \\
\hline
\end{tabular}

${ }^{\text {a }}$ Uniform retail meat identity standards (Industry-Wide Cooperative Meat Identification Standards Committee, 2003).

b Universal product code. 
chuck, and miscellaneous had significantly less $(P<0.05)$ external fat than cuts from the rib and loin. Average fat thickness for cuts from the round and chuck was $0.05 \mathrm{~cm}$ and retail cuts from the rib and loin had $0.11 \mathrm{~cm}$ of external fat. It is economically advantageous for retailers to sell beef steaks from the rib and loin with more external fat because of the value difference between fat left on a steak and that fat that is trimmed off. In order to show the contributions of individual retail cuts to these means, means and standard deviations for fat thickness from retail cuts from the chuck and rib surveyed in the store are found in Table 2, from the loin and round are shown in Table 3, and those from other miscellaneous beef cuts are in Table 4 .

For a majority of the retail cuts represented in the National Nutrient Database, nutrient information is available for cuts with external fat thickness measurements of $1.27 \mathrm{~cm}, 0.6 \mathrm{~cm}, 0.3 \mathrm{~cm}$, and $0.0 \mathrm{~cm}$ (USDA, 2006). However, much of the data in this study shows that, on average, many of the beef cuts at the retail level would have external fat thickness measurements that would lie between $0.3 \mathrm{~cm}$ and $0.0 \mathrm{~cm}$. As a result of this, nutritional information for these products cannot be accurately derived from the data shown in the National Nutrient Database.

\subsection{Separable tissue components}

Retail cuts in this study were dissected into four basic separable components, separable lean, external fat, seam fat, and bone and heavy connective tissue. Data in Tables 5-9 show means and standard deviations for each of the separable components,

Table 13

Means and standard deviations for percentage extractable fat and moisture (separable lean only) for retail cuts from the beef loin

\begin{tabular}{|c|c|c|c|c|c|c|}
\hline \multirow[t]{2}{*}{ Approved URMIS ${ }^{\mathrm{a}}$ name } & \multirow[t]{2}{*}{$\mathrm{UPC}^{\mathrm{b}}$} & \multirow[t]{2}{*}{$n$} & \multicolumn{2}{|c|}{ Extractable fat (\%) } & \multicolumn{2}{|c|}{ Moisture (\%) } \\
\hline & & & Mean & SD & Mean & SD \\
\hline \multicolumn{7}{|l|}{ Beef loin } \\
\hline Top loin steak & 1398 & 21 & 7.74 & 2.86 & 70.39 & 2.05 \\
\hline Top loin steak bnls & 1404 & 53 & 5.49 & 1.99 & 71.67 & 1.69 \\
\hline Tenderloin steak & 1388 & 35 & 4.78 & 1.77 & 73.44 & 1.68 \\
\hline T-bone steak & 1369 & 43 & 6.27 & 1.77 & 71.54 & 1.58 \\
\hline Porterhouse steak & 1330 & 22 & 6.99 & 2.67 & 70.92 & 2.38 \\
\hline Sirloin steak & 1358 & 1 & 5.27 & & 72.81 & \\
\hline Shell sirloin steak & 1346 & 1 & 6.26 & & 70.28 & \\
\hline Ball tip roast & 1307 & 4 & 3.85 & 1.57 & 72.75 & 2.14 \\
\hline Ball tip steak & 1308 & 14 & 4.26 & 1.50 & 72.74 & 1.46 \\
\hline Flap meat steak & 1326 & 1 & 5.70 & & 74.11 & \\
\hline Tri tip roast & 1429 & 12 & 7.57 & 2.98 & 70.99 & 2.41 \\
\hline Tri tip steak & 1430 & 16 & 6.58 & 1.65 & 71.73 & 1.62 \\
\hline Top sirloin rst bnls cap off & 1419 & 1 & 2.50 & & 73.25 & \\
\hline Top sirloin steak bnls & 1422 & 23 & 4.04 & 1.41 & 73.26 & 1.28 \\
\hline Top sirloin stk bnls cap off & 1426 & 22 & 3.67 & 1.21 & 73.36 & 1.06 \\
\hline Top sirloin cap steak bnls & 1421 & 4 & 4.72 & 0.97 & 72.85 & 0.65 \\
\hline Loin average & & 273 & 5.60 & 2.30 & 72.06 & 1.95 \\
\hline
\end{tabular}

${ }^{a}$ Uniform retail meat identity standards (Industry-Wide Cooperative Meat Identification Standards Committee, 2003).

b Universal product code.

Table 14

Means and standard deviations for percentage extractable fat and moisture (separable lean only) for retail cuts from the beef round

\begin{tabular}{|c|c|c|c|c|c|c|}
\hline \multirow[t]{2}{*}{ Approved URMIS ${ }^{a}$ name } & \multirow[t]{2}{*}{$\mathrm{UPC}^{\mathrm{b}}$} & \multirow[t]{2}{*}{$n$} & \multicolumn{2}{|c|}{ Extractable fat (\%) } & \multicolumn{2}{|c|}{ Moisture (\%) } \\
\hline & & & Mean & SD & Mean & SD \\
\hline \multicolumn{7}{|l|}{ Beef round } \\
\hline Steak & 1494 & 1 & 4.49 & & 72.73 & \\
\hline Steak bnls & 1501 & 16 & 2.83 & 1.79 & 74.12 & 1.51 \\
\hline Top round roast & 1455 & 6 & 2.04 & 0.69 & 74.6 & 0.79 \\
\hline Top round roast cap off & 1454 & 7 & 2.72 & 0.86 & 73.44 & 0.60 \\
\hline Top round steak, $1^{\text {st }}$ cut & 1556 & 6 & 3.31 & 2.16 & 73.46 & 1.54 \\
\hline Top round steak & 1553 & 37 & 3.24 & 1.33 & 73.41 & 1.28 \\
\hline Bottom round rump roast & 1519 & 20 & 4.74 & 1.87 & 73.03 & 1.68 \\
\hline Bottom round roast & 1464 & 24 & 4.44 & 1.97 & 72.81 & 1.70 \\
\hline Bottom round steak & 1466 & 27 & 5.24 & 2.38 & 72.41 & 1.78 \\
\hline Eye round roast & 1480 & 24 & 3.30 & 0.94 & 73.96 & 1.10 \\
\hline Eye round steak & 1481 & 28 & 3.07 & 1.01 & 73.79 & 1.11 \\
\hline Bottom round rst (triangle) & 1463 & 2 & 8.79 & 0.71 & 69.98 & 0.26 \\
\hline Tip roast & 1525 & 5 & 3.92 & 1.27 & 75.07 & 1.27 \\
\hline Tip steak & 1527 & 10 & 3.55 & 1.34 & 74.33 & 1.15 \\
\hline Tip roast cap off & 1526 & 3 & 2.87 & 0.56 & 74.72 & 0.59 \\
\hline Tip steak cap off & 1535 & 17 & 3.15 & 1.29 & 74.64 & 0.90 \\
\hline Sirloin tip center steak & 1550 & 7 & 4.44 & 2.33 & 73.58 & 1.58 \\
\hline Sirloin tip center roast & 1549 & 4 & 4.42 & 1.13 & 74.68 & 1.06 \\
\hline Sirloin tip side steak & 1543 & 8 & 2.91 & 0.83 & 74.54 & 0.97 \\
\hline Cubed steak & 1577 & 31 & 3.72 & 1.57 & 73.63 & 1.50 \\
\hline Round average & & 283 & 3.71 & 1.77 & 73.59 & 1.52 \\
\hline
\end{tabular}

${ }^{a}$ Uniform retail meat identity standards (Industry-Wide Cooperative Meat Identification Standards Committee, 2003).

b Universal product code. 
Table 15

Means and standard deviations for percentage extractable fat and moisture (separable lean only) for miscellaneous beef retail cuts

\begin{tabular}{|c|c|c|c|c|c|c|}
\hline \multirow[t]{2}{*}{ Approved URMIS ${ }^{\mathrm{a}}$ name } & \multirow[t]{2}{*}{$\mathrm{UPC}^{\mathrm{b}}$} & \multirow[t]{2}{*}{$n$} & \multicolumn{2}{|c|}{ Extractable fat (\%) } & \multicolumn{2}{|c|}{ Moisture (\%) } \\
\hline & & & Mean & SD & Mean & SD \\
\hline Beef shank cross cuts & 1636 & 40 & 2.97 & 1.25 & 75.51 & 1.22 \\
\hline \multicolumn{7}{|l|}{ Beef Brisket } \\
\hline Whole bnls & 1615 & 3 & 6.24 & 1.29 & 72.96 & 1.19 \\
\hline Point half bnls & 1628 & 1 & 6.57 & & 71.87 & \\
\hline Flat half bnls & 1623 & 14 & 3.90 & 1.79 & 74.66 & 1.76 \\
\hline Middle cut bnls & 1626 & 2 & 5.07 & 0.81 & 74.07 & 0.97 \\
\hline Flat cut bnls & 1622 & 14 & 4.86 & 1.82 & 73.86 & 1.57 \\
\hline Point off bnls & 1629 & 6 & 3.81 & 1.00 & 74.42 & 0.64 \\
\hline Edge cut bnls & 1624 & 1 & 6.64 & & 71.50 & \\
\hline Beef flank steak & 1581 & 38 & 5.72 & 1.92 & 72.77 & 1.65 \\
\hline \multicolumn{7}{|l|}{ Beef plate } \\
\hline Skirt steak bnls & 1607 & 23 & 9.81 & 4.71 & 69.92 & 3.81 \\
\hline Skirt steak rolls bnls & 1611 & 5 & 8.58 & 2.89 & 70.18 & 2.48 \\
\hline Short ribs & 1599 & 7 & 12.13 & 3.17 & 67.99 & 3.33 \\
\hline Spareribs & 1598 & 1 & 12.07 & & 68.40 & \\
\hline Short ribs bnls & 1605 & 8 & 6.43 & 3.49 & 72.89 & 3.11 \\
\hline Short ribs flanken style & 1603 & 10 & 13.61 & 4.03 & 67.69 & 3.04 \\
\hline Beef for stew & 1727 & 42 & 4.26 & 1.50 & 73.75 & 1.54 \\
\hline Beef for stirfry & & 29 & 4.03 & 2.08 & 72.87 & 1.67 \\
\hline Miscellaneous average & & 244 & 5.63 & 3.66 & 72.93 & 2.91 \\
\hline
\end{tabular}

a Uniform retail meat identity standards (Industry-Wide Cooperative Meat Identification Standards Committee, 2003).

b Universal product code.

as well as total separable fat for individual cuts. Table 10 shows least squares means and standard errors for each primal and the miscellaneous beef retail cut category. Cuts from the round had the highest $(P<0.05)$ percentage of separable lean compared to all other primals and categories (Table 10). Cuts from the rib had the lowest $(P<0.05)$ percentage (Table 10$)$. As would be expected, the percentage of total separable fat decreased when the percentage of separable lean increased. Because of this, round cuts also had the lowest percentage of external and seam fat, resulting in the lowest percentage of total separable fat. This is partially because most round cuts are single-muscle cuts, which diminishes the amount of seam fat. Cuts from the rib had the lowest $(P<0.05)$ percentage of separable lean and highest $(P<0.05)$ percentage of total separable fat (Table 10). Specifically, back ribs produced a much lower numeric percentage (34.45\%) for separable lean causing the overall lean percentage mean for rib cuts to be lower than its counterparts (Table 6). Savell et al. (1991) stated that it is expected that cuts from the rib and chuck to have higher percentages of seam fat than cuts from other primals because many of these are multiple muscle cuts. Results from this study support Savell et al. (1991) finding the rib and chuck cuts to have the highest percentages of seam fat. Unlike seam fat, external fat can be removed relatively easily from retail cuts, and after the release of the National Consumer Retail Beef Study (Cross et al., 1986; Savell et al., 1989), retailers made tremendous efforts to decrease the amount of external fat on cuts in the retail case. Innovative fabrication styles are being used more in industry today, and these account for some of the decrease in fat trim levels at retail; however, retailer product specifications have specific external fat thickness requirements for incoming product and may also have contributed to this decrease. Cobiac, Droulez, Leppard, and Lewis (2003) conducted a survey in Australia of retail outlets similar to the present study and the National Beef Market Basket Survey (Savell et al., 1991). Cobiac et al. (2003) stated that there was a wide variation in the percentage of total separable fat in the retail beef cut section. This variation could lead to difficulty in providing accurate nutrient composition data for beef retail cuts.

In general, boneless, closely-trimmed cuts tended to produce a higher percentage of separable lean than others. Additionally, steaks produced a higher percentage of separable lean than roast counterparts because of increased trimming during fabrication.

\subsection{Extractable fat and moisture of separable lean}

Chemical fat and moisture analyses were conducted on the separable lean component obtained from the dissection of each retail cut. Means and standard deviations for the percentages of extractable fat and moisture are presented in Tables 11-16. Table 17 displays the least squares means for percentage extractable fat and moisture of retail cuts from the chuck, rib, loin, and round primals, and other miscellaneous beef cuts. These data follow the same trend reported in the separable component results section with cuts from the round having the lowest percentage of extractable fat and rib cuts generating the highest $(P<0.05)$ percentage (Table 17). Mean extractable fat percentages for nine of the twelve ground beef classifications were lower than what was declared on the package label for fat percentage (Table 16). These results agree with findings from the previous National Beef Market Basket Survey (Savell et al., 1991). Mean percentages for extractable moisture tended to decrease as the percentage of extractable fat increased.

Table 16

Means and standard deviations for percentage extractable fat and moisture for ground beef

\begin{tabular}{|c|c|c|c|c|c|}
\hline \multirow[t]{2}{*}{ Declared lean/fat percentage } & \multirow[t]{2}{*}{$n$} & \multicolumn{2}{|c|}{ Extractable fat (\%) } & \multicolumn{2}{|c|}{ Moisture (\%) } \\
\hline & & Mean & SD & Mean & SD \\
\hline $73 / 27$ & 10 & 22.67 & 3.13 & 60.34 & 2.10 \\
\hline $75 / 25$ & 3 & 23.94 & 1.99 & 59.37 & 2.21 \\
\hline $78 / 22$ & 4 & 17.83 & 3.60 & 63.65 & 2.29 \\
\hline $80 / 20$ & 49 & 17.02 & 2.81 & 64.54 & 2.22 \\
\hline $81 / 19$ & 3 & 22.32 & 1.22 & 60.1 & 0.26 \\
\hline $85 / 15$ & 50 & 13.38 & 2.63 & 67.22 & 2.16 \\
\hline $90 / 10$ & 35 & 8.88 & 2.00 & 71.29 & 1.67 \\
\hline $91 / 9$ & 2 & 8.75 & 1.40 & 71.57 & 0.83 \\
\hline $92 / 8$ & 4 & 7.69 & 0.89 & 71.88 & 1.09 \\
\hline $93 / 7$ & 40 & 8.11 & 3.30 & 71.76 & 3.15 \\
\hline $95 / 5$ & 7 & 4.34 & 1.33 & 74.63 & 1.47 \\
\hline $96 / 4$ & 28 & 6.04 & 2.06 & 72.66 & 1.88 \\
\hline Ground beef average & 235 & 13.41 & 7.06 & 67.42 & 5.57 \\
\hline
\end{tabular}


Table 17

Least squares means $\pm \mathrm{SEM}^{\mathrm{a}}$ for percentage extractable fat and moisture of retail cuts from the chuck, rib, loin, and round primals, and other miscellaneous beef cuts

\begin{tabular}{|c|c|c|c|c|c|c|}
\hline Percentage & Chuck & Rib & Loin & Round & Misc & $P>\mathrm{F}$ \\
\hline Extractable fat & $6.90 \pm 0.15 d$ & $8.61 \pm 0.20 \mathrm{e}$ & $5.60 \pm 0.17 c$ & $3.71 \pm 0.17 a$ & $4.99 \pm 0.19 b$ & $<0.0001$ \\
\hline Extractable moisture & $72.36 \pm 0.12 b$ & $70.00 \pm 0.16 a$ & $72.06 \pm 0.14 b$ & $73.59 \pm 0.13 c$ & $73.36 \pm 0.15 c$ & $<0.0001$ \\
\hline
\end{tabular}

Means within the same row lacking a common letter (a-e) differ $P<0.05$.

a SEM is the standard error for least squares means.

These findings are similar to those reported by Jones, Savell, and Cross (1992b) and Wahrmund (1999).

\section{Conclusions}

Compared to the findings of the previous market basket survey (Savell et al., 1991), it is clear that beef in the current survey had less external fat and separable fat. It is important to continue to conduct market surveys such as these to have the most current information regarding beef retail cut composition available for those entities that need access to these data.

\section{Acknowledgement}

Scientific contribution from Texas AgriLife Research. This project was funded, in part, by The Beef Checkoff.

\section{References}

AOAC (2000). Official methods of analysis (15th ed.). Association of Official Analytica Chemists. Arlington, VA: AOAC

Cobiac, L., Droulez, V., Leppard, P., \& Lewis, J. (2003). Use of external fat width to describe beef and lamb cuts in food composition tables. Journal of Food Composition and Analysis, 16, 133-145.

Cross, H. R., Savell, J. W., \& Francis, J. J. (1986). National consumer retail beef study. Proceedings reciprocal meat conference, 39, 112-116.
Industry-Wide Cooperative Meat Identification Standards Committee. (2003). Uniform retail meat identity standards. Centennial, CO, Cattlemen's Beef Board and National Cattlemen's Beef Association.

Jones, D. K., Savell, J. W., \& Cross, H. R. (1992a). Effects of fat trim on the composition of beef retail cuts - 1. Separate tissue components. Journal of Muscle Foods, 3, 45-56.

Jones, D. K., Savell, J. W., \& Cross, H. R. (1992b). Effects of fat trim on the composition of beef retail cuts -2 . Fat and moisture content of the separable lean. Journal of Muscle Foods, 3, 57-71.

Jones, D. K., Savell, J. W., \& Cross, H. R. (1992c). Effects of fat trim on the composition of beef retail cuts -3 . Cooking yields and fat retention of the separable lean. Journal of Muscle Foods, 3, 73-81.

Savell, J. W., Cross, H. R., Francis, J. J., Wise, J. W., Hale, D. S., Wilkes, D. L., et al (1989). National consumer retail beef study: Interaction of leanness, price, and palatability on consumer acceptance of steaks and roasts of different grades and trimness levels. Journal of Food Quality, 12, 251-274.

Savell, J. W., Harris, J. J., Cross, H. R., Hale, D. S., \& Beasley, L. C. (1991). National beef market basket survey. Journal of Animal Science, 69, 2883-2893.

USDA. (1986). Composition of foods: Beef and beef products - Raw, processed, prepared. Human Nutrition Information Service, United States Department of Agriculture, Agriculture Handbook 8-13.

USDA. (2006). United States Department of Agriculture: Agricultural Research Service. <http://www.ars.usda.gov/main>. Accessed 19.04.06.

Wahrmund, J. L. (1999). Physical and chemical analyses of separable lean of beef retail cuts with different external fat trim levels. M.S. Thesis, Texas A\&M University, College Station.

Wahrmund-Wyle, J. L., Harris, K. B., \& Savell, J. W. (2000a). Beef retail cut composition: 1. Separable tissue components. Journal of Food Composition and Analysis, 13, 233-242.

Wahrmund-Wyle, J. L., Harris, K. B., \& Savell, J. W. (2000b). Beef retail cut composition: 2. Proximate analysis. Journal of Food Composition and Analysis, 13, 243-251. 